\title{
Analysis of 2897 hospitalization events for patients with chronic kidney disease: results from CKD-JAC study
}

\author{
Satoshi limuro ${ }^{1}$ (1) $\cdot$ Tetsuji Kaneko $^{1} \cdot$ Yasuo Ohashi $^{2} \cdot$ Tsuyoshi Watanabe $^{3} \cdot$ Kosaku Nitta $^{4} \cdot$ Tadao Akizawa $^{5}$. \\ Seiichi Matsuo ${ }^{6}$. Enyu Imai $^{7}$. Hirofumi Makino ${ }^{8} \cdot$ Akira Hishida $^{9} \cdot$ for the CKD-JAC Investigators
}

Received: 5 September 2018 / Accepted: 18 March 2019 / Published online: 9 April 2019

(c) The Author(s) 2019

\begin{abstract}
Background Chronic kidney disease is a known risk factor for end-stage renal and cardiovascular diseases. However, data are limited on the causes of hospitalization in patients with chronic kidney disease of maintenance period. This study aimed to aggregate hospitalization data of CKD patients and to determine the high-risk population. In addition, we compared CKD population to general population.

Methods We conducted a post hoc analysis of the chronic kidney disease-Japan cohort study, a multicenter prospective cohort study of 2966 patients with chronic kidney disease with a median 3.9 years of follow-up. We examined the hospitalization reasons and analyzed the risk factors.

Results We found 2897 all-cause hospitalization events (252.3 events/1000 person-years), a hospitalization incidence 17.1fold higher than that in an age- and sex-matched cohort from the general Japanese population. Kidney, eye and adnexa, and heart-related hospital admissions were the most common. All-cause hospitalization increased with chronic kidney disease stage and with the presence of diabetes. Patients with diabetes at enrollment had 345.7 hospitalization events/1000 personyears, which is considerably higher than 196.8 events/1000 person-years for those without diabetes. Survival analysis, using hospitalization as an event, showed earlier all-cause hospitalization with the progression of chronic kidney disease stage and diabetes. Cardiovascular disease hospitalizations were more strongly influenced by diabetes than chronic kidney disease stage. Conclusions Patients with chronic kidney disease and diabetes are highly vulnerable to hospitalization for a variety of diseases. These descriptive data can be valuable in predicting the prognosis of patients with chronic kidney disease.
\end{abstract}

Keywords Diabetes mellitus · Diabetic kidney disease · Cardiovascular disease $\cdot$ Pre-renal replacement therapy $\cdot$ Japanese patient survey 2008

Electronic supplementary material The online version of this article (https://doi.org/10.1007/s10157-019-01730-9) contains supplementary material, which is available to authorized users.

Satoshi Iimuro

siitky@gmail.com

1 Teikyo Academic Research Center, Teikyo University, 2-11-1, Kaga, Itabashi-ku, Tokyo, Japan

2 Department of Integrated Science and Engineering for Sustainable Society, Chuo University, Bunkyo-ku, Tokyo, Japan

3 Japan Organization of Occupational Health and Safety Fukushima Rosai Hospital, Iwaki-city, Fukushima, Japan

4 Department of Nephrology, Tokyo Women's Medical University, Shinjuku-ku, Tokyo, Japan
5 Division of Nephrology, Department of Medicine, Showa University School of Medicine, Shinagawa-ku, Tokyo, Japan

6 Department of Nephrology, Nagoya University, Nagoya, Aichi, Japan

7 Nakayamadera Imai Clinic, Takarazuka, Hyogo, Japan

8 Okayama University, Okayama, Okayama, Japan

9 Yaizu City Hospital, Yaizu, Shizuoka, Japan 


\section{Introduction}

Chronic kidney disease (CKD) is a global public health problem [1]. Multiple cohort studies have been initiated worldwide to investigate its causes and effects [2]. The chronic renal insufficiency cohort (CRIC) study $[3,4]$ in the United States enrolled 4000 participants with CKD, and the resulting data have been used in many publications. On a strong cooperative relationship with the CRIC organizers, Japanese researchers initiated the chronic kidney diseaseJapan cohort (CKD-JAC) study, a multicenter prospective cohort study of Asian patients with stages 3, 4, or 5 CKD living in Japan, aged 20-75 years that monitored patients for 4 years [5, 6]. Data from these studies are being used to determine disease-risk profiles for CKD patients and establish risk factors predicting CKD progression [7, 8].

The CRIC, CKD-JAC, and other cohort studies have clearly established CKD as an independent risk factor for end-stage kidney disease (ESKD), cardiovascular disease (CVD), and all-cause death [4, 7, 9]. Proteinuria, hypertension, diabetes, and dyslipidemia can worsen CKD [10-13], and diabetes is particularly important in countries where it is becoming increasingly common, such as Japan. Diabetic nephropathy is the primary cause of ESKD in Japan, and diabetes accounts for $45 \%$ of all ESKD cases. Chronic glomerulonephritis, which accounted for $60 \%$ of ESKD cases in Japan approximately 30 years ago, is now the etiology in only $20 \%$ of cases [14].

Hospitalization is another concern for CKD patients. Studies on hospitalizations are limited but report a relatively high incidence of hospitalization for arteriovenous shunting, CVD, and infection [15]. In another study, all-cause hospitalization increased with CKD stage [16]. Meanwhile, Japanese medical checkup data have shown that a lower estimated glomerular filtration rate (eGFR) is associated with increased risk of all-cause hospitalization and CVD death [17].

CKD patients are hospitalized not only for CKD, but for diabetes and related diseases. However, there are no reports on CKD patients' hospitalization for other diseases or the contribution of CKD to them.

We aimed to determine the frequency and causes of hospitalization of CKD patients, to elucidate the prognosis using hospitalization as an indicator, and find high-risk population among them.

\section{Materials and methods}

\section{Study design}

CKD-JAC design has been published previously [5]. Inclusion criteria included the absence of polycystic kidney disease, HIV, liver cirrhosis, and cancer, and no history of receiving a transplant, or dialysis. The baseline disease, demographic data, and results of the primary analysis have been published previously [6-8]. The study protocol was approved by the institutional review boards at the institutions involved in the study, and CKD-JAC was conducted in accordance with the Declaration of Helsinki. For this study, formal approval and informed consent were not required as we analyzed previously published data. As for past history, diabetes was defined as $\mathrm{HbA} 1 \mathrm{C}$ values of $6.5 \%$ or higher, taking antidiabetics and/or primary doctors' reporting of with/without diabetes at enrollment. Diabetic nephropathy and glomerulonephritis were defined by primary doctors' reporting regarding main cause for CKD at enrollment. HbA1c was expressed using NGSP.

\section{Hospitalization data}

Hospitalizations were recorded from the enrollment date until the patient began dialysis. Thus, the data reflect only the maintenance period of CKD. The following hospitalization data were collected: (1) date of hospitalization and discharge, (2) diagnoses (maximum three), and (3) treatment (maximum three). Diagnosis and treatment could be entered using the code of dialysis outcomes and practice patterns study [18], but for many hospitalization events, the entries were free-form. For each hospitalization, the primary disease was identified by referring to the diagnosis and treatment. Hospitalizations were classified into 12 main disease groups: [01] infectious diseases, [02] malignant neoplasm, [03] endocrine, nutritional, and metabolic diseases, [04] diseases of the eye and adnexa, [05] diseases of the circulatory system, [06] heart diseases, [07] diseases of the respiratory system, [08] diseases of the digestive tract, [09] hepato-biliary-pancreatic diseases, [10] kidney diseases, [11] other 1 (otorhinolaryngology, dermatology, orthopedic conditions including bone and muscle, urology, obstetrics, and gynecology), and [12] other 2 (benign neoplasm, trauma, emergency, hematologic disease, psychiatric, and neurological). These groups are referred to as CKD-JAC classifications.

In addition to the CKD-JAC classification, diseases were also coded by the Japanese Ministry of Health, Labor and Welfare (MHLW) disease classification codes, based on the 2003 edition of the International Statistical 
Classification of Diseases and Related Health Problems [19]. Using disease classification codes enabled comparisons between CKD-JAC data and other epidemiological data.

Through these processes, for each hospitalization, one main disease name, and CKD-JAC and MHLW disease classification codes were identified. Relationships between the MHLW and CKD-JAC classification codes are shown in Supplementary Table 1.

\section{Control population}

Hospitalization data for a general population comparable to the CKD-JAC population were obtained from the Patient Survey of 2008, published every 3 years by the Japanese MHLW [20], which provides random stratified sampling of patients who use medical care institutions across Japan. We used the 2008 edition because the CKD-JAC research began in 2006 and the next edition was likely affected by the major earthquake in northeast Japan in 2011. To compare the patient survey and CKD-JAC hospitalization data, we matched the patient survey population to the CKD-JAC population using sex, age, and person-years of observation as adjustment factors.

\section{Statistical analyses}

The incidence of hospitalization was expressed as both the total number of hospitalization events and number of events per 1000 person-years. Kaplan-Meier survival analysis was applied for the time to first hospitalization, and survival curves were used to estimate the event-free survival rate. Even when patients had been hospitalized for other diseases, they were not removed from the group "at risk" for the first hospitalization event for ESKD or CVD. For this descriptive analysis of hospitalization, statistical significance was not assessed.

\section{Results}

\section{Baseline demographics and disease-related data}

From 3087 patients enrolled, 121 patients were excluded for some reasons, and finally, 2966 patients were observed for a median of 3.9 years $[7,8]$. The mean age was $60.3 \pm 11.6$ years $(38 \%$ women). CKD stages at enrollment were stage $3 \mathrm{a}$ [306 patients (10\%)], stage 3b [1045 (35\%)], stage 4 [1149 (39\%)], stage 5 [466 (16\%)] [7]. Diabetic patients were 1117 (62.3\%), especially those with DM nephropathy were 612 (20.6\%). Glomerulonephritis patients were $946(31.9 \%)$. A total of $11,484.55$ person-years were observed.

\section{Frequency of hospitalization for each disease}

We observed 2897 all-cause hospitalization events (252.3 hospitalizations/1000 person-years, Table 1a). Supplemental Supplementary Table 1 shows specifics of each disease classification in the CKD-JAC classification. The most common reason for hospitalization was [10] kidney disease (86.1 events/1000 person-years, Supplementary Table 2). A total of 989 renal hospitalizations were observed mostly due to $\operatorname{ESKD~}(n=758)$, such as hospitalization to create a vascular access or start dialysis (Table 2). CKD stage was strongly associated with the number of ESKD hospitalizations: 4.9 events/1000 person-years for stage 3a CKD and 230.6 events/ 1000 person-years for stage 5 CKD.

The next most common reasons for hospitalization were CVD-related issues ([05] circulatory system and [06] heart), [01] infectious disease, and [02] malignant neoplasm, at 37.3, 19.7, and 17.7 events/1000 person-years, respectively. We observed $428 \mathrm{CVD}$-related hospitalization events: ischemic heart disease, 13.1 events/1000 personyears; stroke (cerebral infarction and other cerebrovascular diseases) 5.8 events/1000 person-years (Supplementary Table 2). The most common infection in group [01] was pneumonia. The most common type of [02] malignant neoplasm was gastrointestinal (Supplementary Table 3). [03] Endocrine, nutritional, and metabolic diseases accounted for 16.6 events/1000 person-years, with diabetes mellitus accounting for 9.3 events/1000 person-years.

\section{Sex differences in hospitalization rates on CKD-JAC}

All-cause hospitalization was slightly higher in men (271.7 hospitalization events/1000 person-years) than women (221.4 events/1000 person-years). Hospitalizations for [07] respiratory system diseases and [06] heart were 2.1-fold and 2.0-fold more frequent in men, respectively. Hospitalization rates owing to [02] malignant neoplasm, [05] circulatory system, [09] hepato-biliary-pancreatic, and [10] kidney diseases were 1.7-fold, 1.7-fold, 1.5-fold, and 1.4-fold higher in men, respectively (Table 1a).

\section{Differences in hospitalization rates by CKD stage}

All-cause hospitalization increased with CKD stage; stage 3a had 171.0 events/1000 person-years, increasing to 388.4 events/1000 person-years at stage 5 . Hospital admissions for [10] kidney disease increased sharply at stage 5 (Fig. 1a). For [05] circulatory system, [06] heart, and [08] digestive tract, hospitalizations increased from stage $3 a$ to 4 and decreased in stage 5 (Table 1b). For most other diseases, the number of hospitalizations was consistent across CKD stages (Fig. 1). Patients were hospitalized for a variety of diseases at CKD stages $3 \mathrm{a}$ and $3 \mathrm{~b}$, but at stages 4 and 5 , there 
Table 1 CKD-JAC classification for all hospitalizations (per 1000 patients)

\begin{tabular}{llll}
\hline CKD-JAC classification & All & \multicolumn{1}{c}{ Male } & Female \\
& Per 1000 person-years & \\
\hline
\end{tabular}

(a) All study subjects/subjects grouped by sex

01. Infectious diseases $\quad 19.7$

02. Malignant neoplasm

$\begin{array}{llll}19.7 & 20.7 & 18.0 & 1.2\end{array}$

03. Endocrine, nutritional and metabolic diseases

$17.7 \quad 21.0$

$12.4 \quad 1.7$

04. Diseases of the eye and adnexa

16.6

16.3

$17.1 \quad 1.0$

05 . Diseases of the circulatory system

$15.0 \quad 14.9$

$15.1 \quad 1.0$

06. Heart diseases

12.7

14.9

$8.8 \quad 1.7$

07 . Diseases of the respiratory system

24.6

30.5

15.1

$3.7 \quad 4.7$

08. Diseases of digestive tract

$15.7 \quad 15.3$

09. Hepato-biliary-pancreatic diseases

10. Diseases of kidney

$\begin{array}{ll}6.4 & 7.2\end{array}$

11. Others 1

$86.1 \quad 97.3$

12. Others 2

22.8

Total

11.3

252.3

2.2

$\begin{array}{ll}2.2 & 2.1 \\ 16.2 & 0.9\end{array}$

$\begin{array}{lll}1.3 & 16.2 & 0.9 \\ .2 & 4.9 & 1.5\end{array}$

$\begin{array}{lll}97.3 & 68.4 & 1.4\end{array}$

$\begin{array}{lll}18.2 & 30.1 & 1.4 \\ 10.2 & 0.6\end{array}$

CKD-JAC classification

\begin{tabular}{lll} 
CKD stage & & \\
\hline $3 \mathrm{a} \quad 3 \mathrm{~b}$ & 4 & 5 \\
Per 1000 person-years & &
\end{tabular}

(b) Subjects grouped by CKD stage

01. Infectious diseases

18.9

13.2

02. Malignant neoplasm

19.7

14.8

04. Diseases of the eye and adnexa

05. Diseases of the circulatory system

06. Heart diseases

07. Diseases of the respiratory system

08. Diseases of the digestive tract

09. Hepato-biliary-pancreatic diseases

10. Kidney diseases

11. Other 1

12. Other 2

Total

9.9

17.3

7.4

6.6

3.3

18.9

26.3

14.8

171.0

17.7
22.5
13.4
14.4
12.2
29.3
3.4
17.0
8.2
31.6
23.5
10.3
203.6

22.1

17.6

18.3

16.7

15.6

21.9

3.6

19.8

6.3

92.4

23.7

11.5

269.4
18.7

9.0

18.1

12.0

8.4

25.3

2.4

7.8

4.2

255.3

16.3

10.8

388.4

CKD chronic kidney disease, CKD-JAC chronic kidney disease-Japan cohort

was a sharp increase in the percentage of patients hospitalized for [10] kidney diseases (Fig. 2a).

\section{Effects of underlying disease}

All-cause hospitalization was higher in diabetic patients than in those who were diabetes-free at enrollment (345.7 events/1000 person-years vs. 196.8 events/1000 personyears; Table 3). Within the subgroup of patients with diabetic nephropathy, total hospitalizations reached 381.2 events/1000 person-years. The classifications most affected by diabetes were [03] endocrine, nutritional, and metabolic, [06] heart [04] eye and adnexa, and [10] kidney. Patients who got CKD mainly from glomerulonephritis could keep relatively good conditions rather than not only those with diabetes but also those with other underlying diseases (175.2 events/1000 person-years; Table 3).

When we aggregated the hospitalizations without [10] kidney disease (mainly for ESKD), the diabetes group had nearly twofold more hospitalizations than the non-diabetes group (232.1 events/1000 person-years vs. 127.0 events/1000 person-years). The diabetes group had higher hospitalization rates for [03] endocrine, nutritional, and metabolic and [04] eye and adnexa (mostly diabetic retinopathy). Even when we adjusted for those classifications, diabetic patients had more hospitalizations, even for conditions unrelated to diabetes (172.9 vs. 111.7 hospitalization events). The greatest difference between the two groups was for [06] heart disease. 
Table 2 Hospitalization for kidney diseases in patients grouped by CKD disease stage

\begin{tabular}{|c|c|c|c|c|c|c|}
\hline & & \multicolumn{5}{|l|}{ CKD stage } \\
\hline & & All & $3 a$ & $3 b$ & 4 & 5 \\
\hline c-1402 & Nephrotic syndrome & $9(0)$ & $0(0)$ & $7(0)$ & $2(0)$ & $0(0)$ \\
\hline c-1403 & Other glomerular diseases & $47(0)$ & $11(0)$ & $26(0)$ & $8(0)$ & $2(0)$ \\
\hline c-1404 & Renal tubulo-interstitial diseases & $8(0)$ & $0(0)$ & $4(0)$ & $3(0)$ & $1(0)$ \\
\hline c-1405 & Chronic renal failure & $848(758)$ & $6(6)$ & $76(63)$ & $357(304)$ & 409 (380) \\
\hline c-1406 & Other renal failure & $77(5)$ & $6(0)$ & $19(0)$ & $40(2)$ & $12(3)$ \\
\hline Total & & 989 & 23 & 132 & 410 & 424 \\
\hline Per 1000 person-years & & 86.1 & 18.9 & 31.6 & 92.4 & 255.3 \\
\hline Hospitalization not for ESKD & & 231 & 17 & 69 & 104 & 41 \\
\hline Per 1000 person-years & & 20.1 & 14.0 & 16.5 & 23.4 & 24.7 \\
\hline Hospitalization for ESKD & & 758 & 6 & 63 & 306 & 383 \\
\hline Per 1000 person-years & & 66.0 & 4.9 & 15.1 & 69.0 & 230.6 \\
\hline
\end{tabular}

The CKD-JAC Kidney diseases classification [10] includes 989 events of the disease classification code a-1401 (glomerular disease, renal tubulo-interstitial disease, and renal failure) from the Ministry of Health, Labour and Welfare (MHLW) major classifications of disease. These specifics were expressed using the MHLW disease classification's sub-classification code. The numbers within parentheses are the number of hospitalizations related to end-stage kidney disease

$C K D$ chronic kidney disease, ESKD end-stage kidney disease, $C K D$-JAC chronic kidney disease-Japan cohort

However, even without that classification, there were 128.1 hospitalization events/1000 person-years in the diabetes group and 99.2 in the non-diabetes group.

\section{Hospitalization duration}

Median durations of each disease categories were from about 12 days to 14 days. There were little differences between male and female, CKD stages and four categories of underlying diseases (Supplementary Fig. 1).

\section{Comparison to a control population}

The control population from the patient survey of 2008 [20] had 14.8 hospitalization events/1000 person-years (Table 4a), higher in men (16.8 events/1000 person-years) than women (11.4 events/1000 person-years), of which tendency was also observed in CKD-JAC.

The CKD-JAC population had 17.1-fold more all-cause hospitalization events than controls. Hospitalizations were markedly higher in the CKD-JAC population for all individual conditions, especially the [10] kidney (218.0-fold) and [04] eye and adnexa (100.5-fold) classifications. However, even when we removed the classifications closely related to CKD ([10] kidney, [03] endocrine, nutritional and metabolic, and [04] eye and adnexa) from the all-cause hospitalization data, hospitalizations were still 9.7-fold more frequent in the CKD-JAC population than in controls. This trend was relatively consistent in both men and women (Table 4b).

\section{Time to the first hospitalization based on CKD stage}

\section{All-cause hospitalization}

Survival curves were similar for CKD stages $3 a$ and $3 b$. For stage 4 , the number of hospitalizations began to rise after the first year. The stage 5 survival curve diverged immediately. During the first year of follow-up, only $63 \%$ of stage 5 patients were still not hospitalized, compared to $86 \%$ of stage 3 a patients, a difference of $23 \%$. By the fourth year, that difference had increased to $39 \%$ [22\% (stage 5) vs. $60 \%$ (stage 3a); Fig. 3a].

Diabetes profoundly affected hospitalization-free survival (Fig. 3b). In patients grouped by CKD stage and diabetes mellitus (DM + or DM-), hospitalization occurred earliest in stage $5 \mathrm{DM}+$ patients, followed by stage $5 \mathrm{DM}-$, next stage $4 \mathrm{DM}+$, and then stage $3 \mathrm{~b} \mathrm{DM}+$ or stage $3 \mathrm{a} \mathrm{DM}+$. Firstyear hospitalization-free rates for these groups were $51 \%$, $72 \%, 75 \%, 80 \%$, and $81 \%$, respectively. The time to the first hospitalization was longest for stage $3 \mathrm{a} \mathrm{DM}$ - patients, stage 3b DM - patients, and stage $4 \mathrm{DM}$ - patients (Fig. 3c, d).

\section{Hospitalization for CVD}

Survival analysis was applied to analyze the time to first hospitalization for sub-classifications [a-0902] ischemic heart diseases, [a-0903] other heart diseases, [a-0904] cerebral infarction, and [a-0905] other cerebrovascular diseases in 
Fig. 1 Hospitalization for each CKD-JAC classification by

CKD stage. a Events per 1000 person-years with all clas-

sifications. b Events per 1000

person-years with [10] kidney diseases excluded [11]. Other one includes otorhinolaryngology, dermatology, orthopedic conditions including bone and muscle, urology, obstetrics, and gynecology [12]; other two include benign neoplasm, trauma, emergency, hematologic disease, psychiatric, and neurological. $C K D-J A C$ chronic kidney disease-Japan cohort; $C K D$ chronic kidney disease, $3 a$ CKD stage $3 a, 3 b$ CKD stage 3b, 4 CKD stage 4, 5 CKD stage 5 (a) 300 250 200 150 100 50

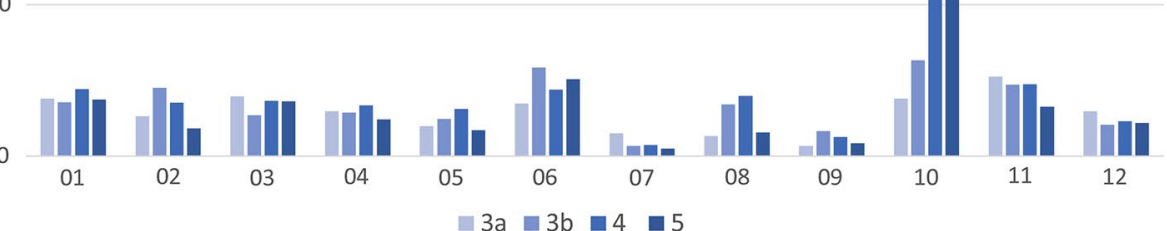

(b) 35

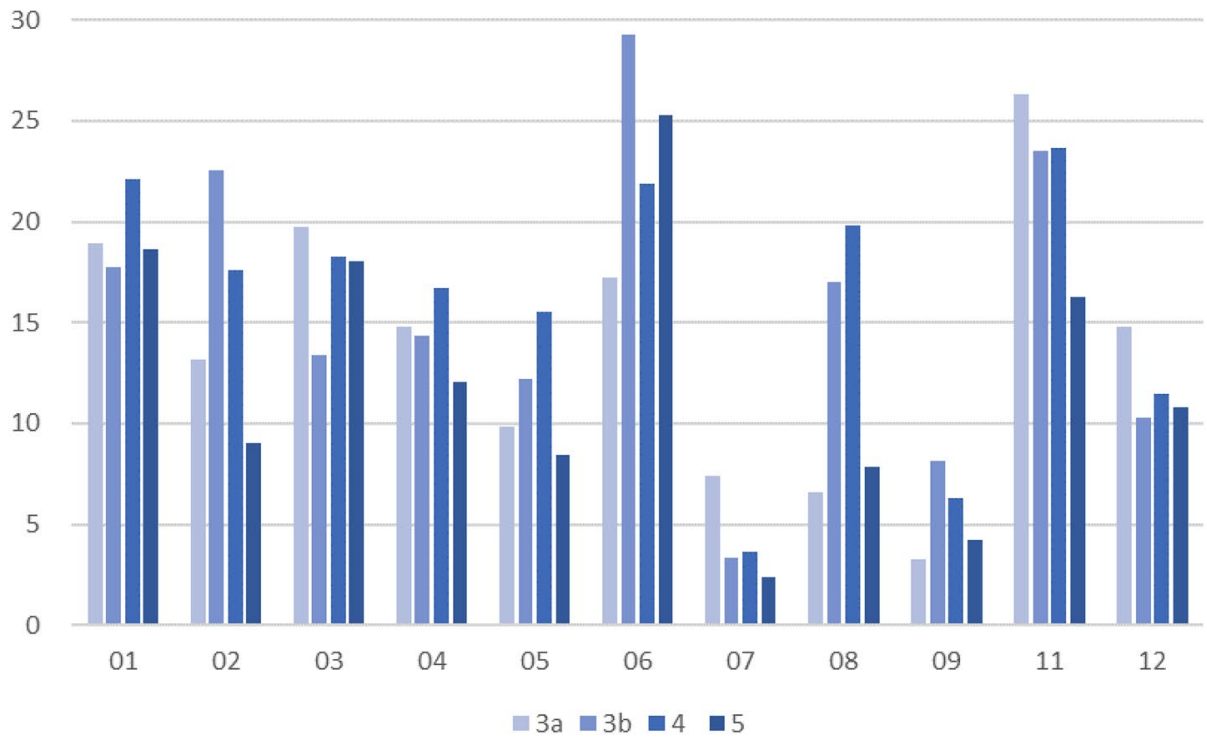

CVD-related issues ([05] circulatory system). After 4 years, hospitalization rates remained low at each stage (Fig. 4a). When we grouped the patients by CKD stage and DM status, diabetes was a clear risk factor for CVD-related hospitalization (Fig. 4b, c). 
Fig. 2 The percentage of hospitalizations attributable to each disease classification. a Patients at different CKD stages with all classifications. b Patients at different CKD stages with [10] kidney diseases excluded. $C K D$ Chronic kidney disease, $3 a$ CKD stage $3 a, 3 b$ CKD stage 3b, 4 CKD stage 4, 5 CKD stage 5 (a)

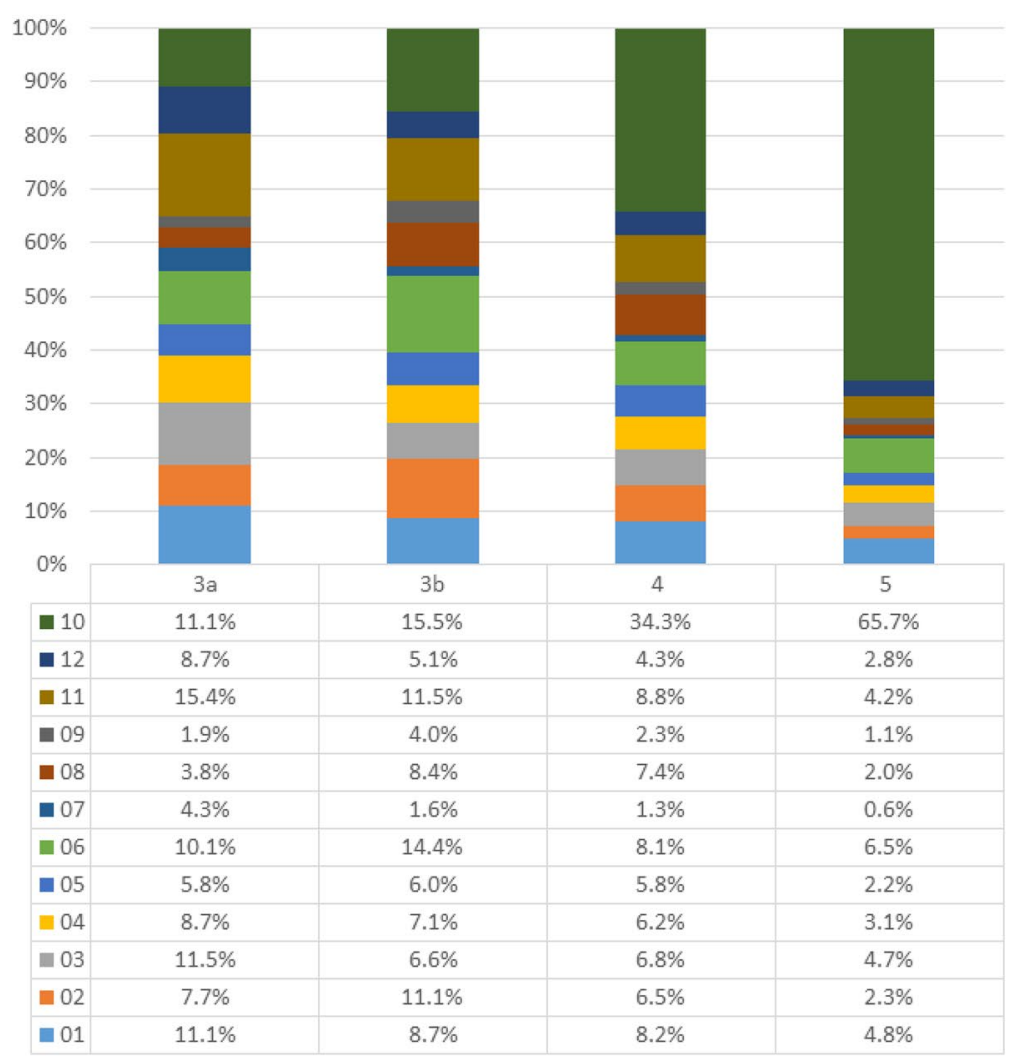

(b)

\begin{tabular}{|c|c|c|c|c|}
\hline $90 \%$ & & & & \\
\hline $80 \%$ & & & & \\
\hline $70 \%$ & & & & \\
\hline $60 \%$ & & & & \\
\hline $50 \%$ & & & & \\
\hline 5070 & & & & \\
\hline $40 \%$ & & & & \\
\hline $30 \%$ & & & & \\
\hline $20 \%$ & & & & \\
\hline $10 \%$ & & & & \\
\hline $0 \%$ & $3 a$ & $3 b$ & 4 & 5 \\
\hline घ12 & $9.7 \%$ & $6.8 \%$ & $7.6 \%$ & $7.1 \%$ \\
\hline a 11 & $17.3 \%$ & $15.4 \%$ & $15.6 \%$ & $10.7 \%$ \\
\hline ש 09 & $2.2 \%$ & $4.7 \%$ & $3.6 \%$ & $3.2 \%$ \\
\hline - 08 & $4.3 \%$ & $9.9 \%$ & $11.2 \%$ & $5.9 \%$ \\
\hline घ 07 & $4.9 \%$ & $2.0 \%$ & $2.0 \%$ & $1.8 \%$ \\
\hline 06 & $11.3 \%$ & $17.0 \%$ & $12.4 \%$ & $19.0 \%$ \\
\hline =05 & $6.5 \%$ & $7.1 \%$ & $8.8 \%$ & $6.3 \%$ \\
\hline 04 & $9.7 \%$ & $8.4 \%$ & $9.4 \%$ & $9.0 \%$ \\
\hline$=03$ & $13.0 \%$ & $7.8 \%$ & $10.3 \%$ & $13.6 \%$ \\
\hline 02 & $8.6 \%$ & $13.1 \%$ & $9.9 \%$ & $6.8 \%$ \\
\hline 01 & $12.4 \%$ & $10.3 \%$ & $12.5 \%$ & $14.0 \%$ \\
\hline
\end{tabular}


Table 3 Hospitalization events for subjects grouped by underlying disease status

\begin{tabular}{|c|c|c|c|c|c|c|}
\hline \multirow[t]{3}{*}{ CKD-JAC classification } & \multirow[t]{2}{*}{$\mathrm{DM}(-)$} & \multirow[t]{2}{*}{$\mathrm{DM}(+)$} & \multicolumn{2}{|l|}{$\mathrm{DM}(-)$} & \multicolumn{2}{|l|}{$\mathrm{DM}(+)$} \\
\hline & & & Others & $\begin{array}{l}\text { Glomerulo- } \\
\text { nephritis }\end{array}$ & $\begin{array}{l}\text { DM nephropa- } \\
\text { thy }(-)\end{array}$ & $\begin{array}{l}\text { DM } \\
\text { nephropa- } \\
\text { thy }(+)\end{array}$ \\
\hline & \multicolumn{6}{|c|}{ Per 1000 person-years } \\
\hline 01. Infectious diseases & 17.6 & 23.1 & 21.5 & 14.0 & 27.3 & 19.5 \\
\hline 02. Malignant neoplasm & 16.7 & 19.4 & 21.2 & 12.4 & 21.8 & 17.4 \\
\hline 03. Endocrine, nutritional and metabolic diseases & 6.7 & 33.4 & 8.6 & 4.8 & 18.7 & 46.0 \\
\hline 04. Diseases of the eye and adnexa & 8.6 & 25.7 & 8.3 & 8.9 & 18.7 & 31.7 \\
\hline 05. Diseases of the circulatory system & 11.0 & 15.7 & 14.9 & 7.3 & 13.2 & 17.8 \\
\hline 06. Heart diseases & 12.5 & 44.9 & 17.5 & 7.8 & 34.4 & 53.8 \\
\hline 07. Diseases of the respiratory system & 2.8 & 5.4 & 4.0 & 1.6 & 5.6 & 5.2 \\
\hline 08. Diseases of digestive tract & 15.7 & 15.7 & 22.1 & 9.7 & 18.7 & 13.0 \\
\hline 09. Hepato-Biliary-Pancreatic diseases & 5.6 & 7.7 & 5.4 & 5.7 & 10.6 & 5.2 \\
\hline 10. Diseases of kidney & 69.8 & 113.6 & 64.5 & 74.8 & 91.1 & 132.9 \\
\hline 11. Others 1 & 19.7 & 28.0 & 20.9 & 18.6 & 32.9 & 23.9 \\
\hline 12. Others 2 & 10.3 & 13.1 & 10.9 & 9.7 & 11.1 & 14.8 \\
\hline Total & 196.8 & 345.7 & 219.8 & 175.2 & 304.2 & 381.2 \\
\hline
\end{tabular}

DM (-) and DM (+) at enrollment, and DM (-)/glomerulonephritis, DM (-)/other, DM (+)/diabetic nephropathy $(-)$ and DM (+)/diabetic nephropathy (+) at enrollment

$D M$ diabetes mellitus; CKD-JAC chronic kidney disease-Japan cohort

\section{Discussion}

In this study, we used data from nearly 4 years of followup on 2966 participants to profile risk factors and underlying causes of hospitalization in CKD patients. With 2897 hospitalization events ( 252.3 events/1000 person-years), we have strong data supporting a few important findings in this vulnerable patients.

These data demonstrate that CKD patients are at high risk of hospitalization for many diseases. All-cause hospitalization was 17.1-fold more common in CKD-JAC patients than in the control cohort. CKD is a risk factor for ESKD and CVD, and the high hospitalization rate for the [10] kidney diseases (86.1 events/1000 person-years) and [06] heart (24.6 events/1000 person-years) classifications is consistent with previous findings $[7,8,15]$. However, the magnitude of that effect in this study was greater than expected: a 218.0fold higher risk for [10] kidney diseases and 46.3-fold higher risk for [06] heart diseases than in the control cohort. Hospitalizations were also more frequent for diseases of the [04] eye and adnexa (100.5-fold) and [03] endocrine, nutritional, and metabolic diseases (39.9-fold). Both classifications are related to diabetes, which is a major underlying disease in CKD. Even after we removed [04] eye and adnexa [03] endocrine, nutritional, and metabolic, as well as [10] kidney diseases, in which ESKD plays a major role, CKD-JAC hospitalizations were still substantially more frequent (9.7-fold) than hospitalizations in controls. This indicates that CKD is a risk factor for many diseases, and CKD patients are highly vulnerable to many conditions that may require hospitalization. This analysis provides a unique comprehensive profile of all kinds of hospitalizations.

Our study also provides valuable data on the effects of CKD stage on hospitalization. Hospitalizations for [10] kidney disease increased dramatically with CKD stage, and hospitalizations for [05] circulatory system and [06] heart diseases were greater at CKD stage 4 than at stage $3 \mathrm{a}$ (Fig. 1b). Previous research has clearly shown that CKD stages 3 through 5 are independent predictors of ESKD and CVD [16, 21-24]. Our data confirm these findings, except for decreased hospitalizations at stage 5 for [05] circulatory system and [06] heart diseases. That may be because only the main disease names from hospitalization data including maximum three diagnoses were analyzed. Competing risk analysis will be done in the future.

We were able to investigate potential risk of underlying diseases on hospitalization. All-cause hospitalization was approximately 1.8 times higher in diabetic patients (345.7 events/1000 person-years in the DM + group vs. 196.8 in the DM - group). Among those with diabetes, hospitalizations were even more frequent in the subgroup of patients diagnosed with diabetic nephropathy (381.2 events/1000 personyears). Results were similar even after excluding disease classifications closely related to diabetes. Clearly, diabetes affects hospitalization for many diseases, both related and unrelated to diabetes. In the group previously diagnosed with 
Table 4 Disease classifications for hospitalization in the control population

\begin{tabular}{|c|c|c|c|c|c|c|}
\hline \multirow[t]{2}{*}{ CKD-JAC classification } & \multicolumn{3}{|c|}{ Control } & \multirow{2}{*}{\multicolumn{2}{|c|}{ CKD-JAC }} & \multirow{2}{*}{$\begin{array}{l}\text { CKD- } \\
\text { JAC/ } \\
\text { control }\end{array}$} \\
\hline & All & Male & Female & & & \\
\hline \multicolumn{7}{|c|}{ (a) Data on the control cohort and comparison between the CKD-JAC and control populations } \\
\hline 01. Infectious diseases & 0.7 & 0.8 & 0.4 & \multicolumn{2}{|c|}{19.7} & 29.1 \\
\hline 02. Malignant neoplasm & 2.2 & 2.7 & 1.3 & \multicolumn{2}{|c|}{17.7} & 8.1 \\
\hline 03. Endocrine, nutritional, and metabolic diseases & 0.4 & 0.5 & 0.3 & \multicolumn{2}{|c|}{16.6} & 39.9 \\
\hline 04. Diseases of the eye and adnexa & 0.1 & 0.1 & 0.2 & \multicolumn{2}{|c|}{15.0} & 100.5 \\
\hline 05. Diseases of the circulatory system & 2.0 & 2.5 & 1.3 & \multicolumn{2}{|c|}{12.7} & 6.3 \\
\hline 06. Heart diseases & 0.5 & 0.7 & 0.3 & \multicolumn{2}{|c|}{24.6} & 46.3 \\
\hline 07. Diseases of the respiratory system & 0.3 & 0.4 & 0.1 & \multicolumn{2}{|c|}{3.7} & 13.6 \\
\hline 08. Diseases of the digestive tract & 0.5 & 0.6 & 0.3 & \multicolumn{2}{|c|}{15.7} & 32.0 \\
\hline 09. Hepato-biliary-pancreatic diseases & 0.3 & 0.4 & 0.2 & \multicolumn{2}{|c|}{6.4} & 18.6 \\
\hline 10. Kidney diseases & 0.4 & 0.5 & 0.3 & \multicolumn{2}{|c|}{86.1} & 218.0 \\
\hline 11. Other 1 & 1.7 & 1.6 & 1.9 & \multicolumn{2}{|c|}{22.8} & 13.5 \\
\hline 12. Other 2 & 5.6 & 6.1 & 4.5 & \multicolumn{2}{|c|}{11.3} & 2.0 \\
\hline Total & 14.8 & 16.8 & 11.4 & \multicolumn{2}{|c|}{252.3} & 17.1 \\
\hline \multirow[t]{2}{*}{ CKD-JAC classification } & \multicolumn{3}{|l|}{ Female } & \multicolumn{3}{|l|}{ Male } \\
\hline & CKD-JAC & Control & $\mathrm{JAC} / \mathrm{ctrl}$ & CKD-JAC & Control & $\begin{array}{l}\text { JAC/ } \\
\text { ctrl }\end{array}$ \\
\hline \multicolumn{7}{|c|}{ (b) Comparisons between female and male subjects in the CKD-JAC and control populations } \\
\hline 01. Infectious diseases & 18.0 & 0.4 & 42.2 & 20.7 & 0.8 & 25.0 \\
\hline 02. Malignant neoplasm & 12.4 & 1.3 & 9.5 & 21.0 & 2.7 & 7.8 \\
\hline 03. Endocrine, nutritional, and metabolic diseases & 17.1 & 0.3 & 49.7 & 16.3 & 0.5 & 35.4 \\
\hline 04. Diseases of the eye and adnexa & 15.1 & 0.2 & 99.0 & 14.9 & 0.1 & 101.4 \\
\hline 05. Diseases of the circulatory system & 8.8 & 1.3 & 6.7 & 15.2 & 2.5 & 6.2 \\
\hline 06. Heart diseases & 15.1 & 0.3 & 50.0 & 30.5 & 0.7 & 45.6 \\
\hline 07. Diseases of the respiratory system & 2.2 & 0.1 & 15.8 & 4.7 & 0.4 & 13.2 \\
\hline 08. Diseases of the digestive tract & 16.2 & 0.3 & 49.1 & 15.3 & 0.6 & 26.1 \\
\hline 09. Hepato-biliary-pancreatic diseases & 4.9 & 0.2 & 20.4 & 7.2 & 0.4 & 17.9 \\
\hline 10. Kidney diseases & 68.4 & 0.3 & 243.6 & 97.3 & 0.5 & 209.3 \\
\hline 11. Other 1 & 30.1 & 1.9 & 15.8 & 18.2 & 1.6 & 11.6 \\
\hline 12. Other 2 & 13.0 & 4.5 & 2.9 & 10.2 & 6.1 & 1.7 \\
\hline Total & 221.4 & 11.4 & 19.4 & 271.7 & 16.8 & 16.1 \\
\hline
\end{tabular}

With data from the Ministry of Health, Labour and Welfare Patient Survey of 2008 and age, sex, andobservation time as adjustment factors, a control cohort from the general population was created tomatch the CKD-JAC group

CKD-JAC Chronic kidney disease-Japan Cohort, ctrl control

glomerulonephritis, the number of hospitalization events was low for most diseases except those in [10] kidney. These findings demonstrate that the disease underlying CKD has important prognostic value and the subpopulation of patients with diabetic nephropathy is at particularly high risk.

Survival analysis also illustrated the major impact of diabetes on CKD. The time to first hospitalization was shorter in patients with a higher CKD stage and in those with diabetes. If we evaluate all-cause hospitalizations, the time to first hospitalization was shorter in patients with CKD stage $3 \mathrm{~b}$ and diabetes than those with CKD stage 4 without diabetes. For CVD-related issues, diabetes had an unexpectedly strong effect on the survival time. The time to CVD-related hospitalization was shorter in diabetic patients than in patients who were diabetes-free, across all CKD stages.

This study had some limitations. First, at CKD stage 5, we expected an increase in hospitalizations related to renal diseases. These patients may have been hospitalized for other diseases, but the diagnoses could be obscured by the primary disease name. To address this, we plan a future investigation of competing risks. Second, there is a problem to be considered whether CKD-JAC data had an appropriate representativeness of CKD patients in Japan. Our data were from large leading hospitals that have nephrologists in residence, but 
(a)

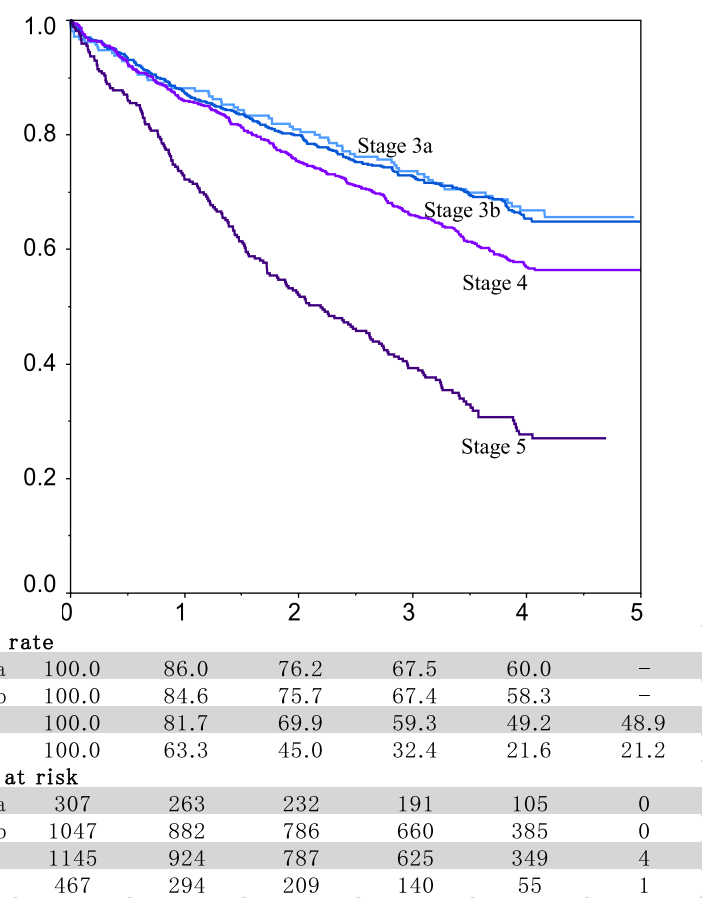

(c)

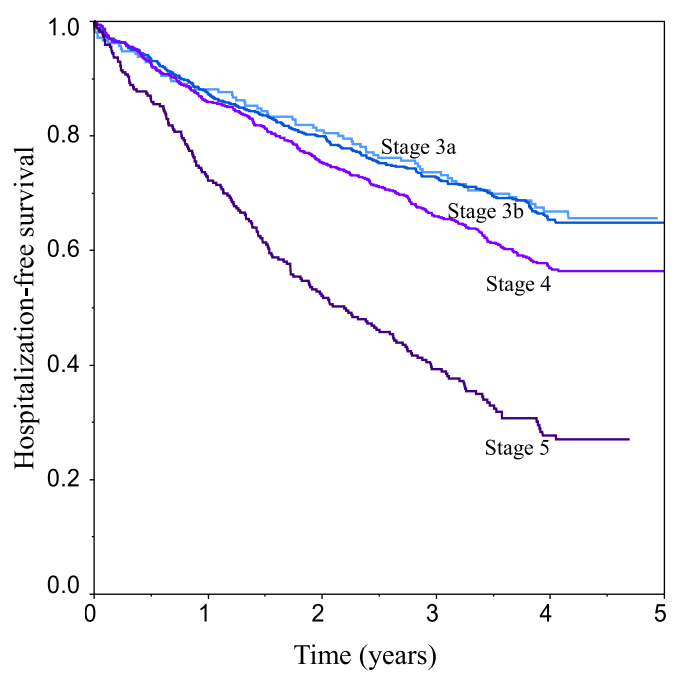

Survival rate

\begin{tabular}{|c|c|c|c|c|c|c|}
\hline \multicolumn{7}{|l|}{ 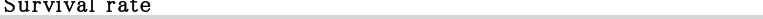 } \\
\hline Stage 3a & 100.0 & 88.1 & 81.0 & 73.7 & 66.8 & - \\
\hline Stage $3 b$ & 100.0 & 87.4 & 80.0 & 72.8 & 65.4 & - \\
\hline Stage 4 & 100.0 & 85.9 & 75.3 & 66.0 & 56.9 & 56.4 \\
\hline Stage 5 & 100.0 & 72.2 & 52.1 & 39.3 & 27.7 & - \\
\hline \multicolumn{7}{|c|}{ Number at risk } \\
\hline Stage $3 a$ & 211 & 185 & 169 & 143 & 81 & 0 \\
\hline Stage $3 b$ & 666 & 578 & 528 & 454 & 282 & 0 \\
\hline Stage 4 & 701 & 594 & 517 & 422 & 245 & 3 \\
\hline Stage 5 & 271 & 194 & 140 & 97 & 42 & 0 \\
\hline
\end{tabular}

Fig. 3 Kaplan-Meier survival curves for the first hospitalization due to any cause. a CKD stage. b DM status. $\mathbf{c}$ CKD stage and DM (-). d CKD stage and DM (+) [11]. Other one includes otorhinolaryngology, dermatology, orthopedic conditions including bone and muscle,

the control data were obtained from a survey of hospitals of all sizes, not necessarily with nephrologists in residence. The results of this comparison may reflect not only differences in (b)

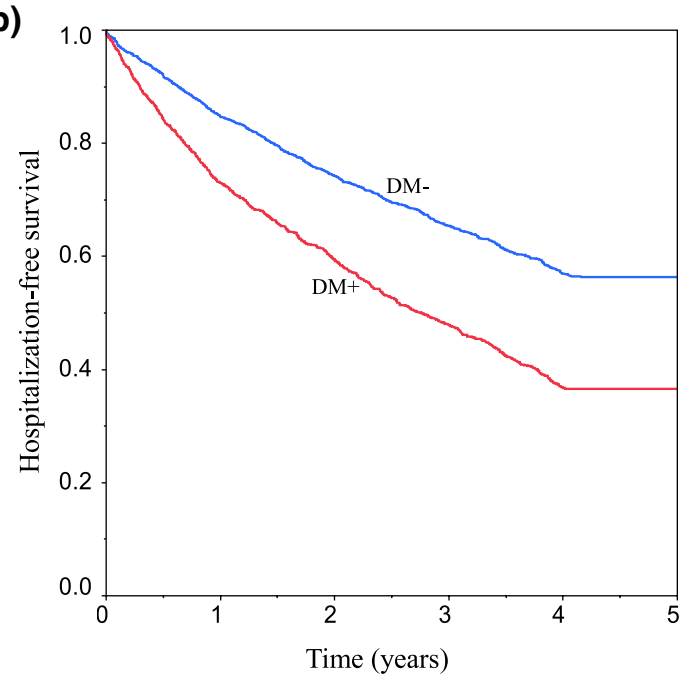

\begin{tabular}{|c|c|c|c|c|c|c|}
\hline \multicolumn{7}{|c|}{ Survival rate } \\
\hline DM- & 100.0 & 84.7 & 74.2 & 65.4 & 57.0 & 56.4 \\
\hline $\mathrm{DM}^{+}$ & 100.0 & 73.0 & 59.5 & 47.9 & 36.7 & 36.6 \\
\hline \multicolumn{7}{|c|}{ Number at risk } \\
\hline DM- & 1849 & 1551 & 1354 & 1116 & 650 & 3 \\
\hline $\mathrm{DM}+$ & 1117 & 812 & 660 & 500 & 244 & 2 \\
\hline
\end{tabular}

(d)

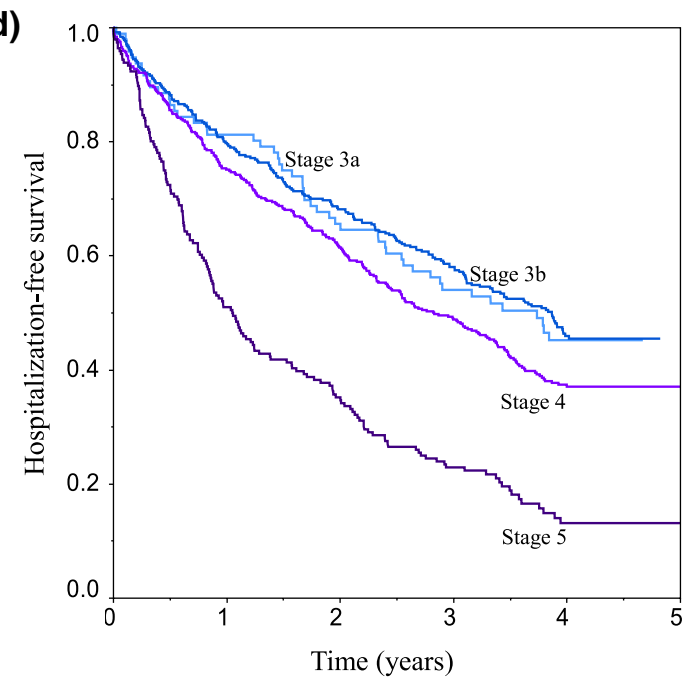

\begin{tabular}{|c|c|c|c|c|c|c|}
\hline \multicolumn{7}{|c|}{ Survival rate } \\
\hline Stage $3 a$ & 100.0 & 81.3 & 65.6 & 54.0 & 45.2 & - \\
\hline Stage $3 b$ & 100.0 & 79.8 & 68.2 & 58.1 & 46.0 & - \\
\hline Stage 4 & 100.0 & 75.1 & 61.5 & 48.9 & 37.1 & 37.1 \\
\hline Stage 5 & 100.0 & 51.0 & 35.2 & 22.9 & 13.2 & 13.2 \\
\hline \multicolumn{7}{|c|}{ Number at risk } \\
\hline Stage $3 a$ & 96 & 78 & 63 & 48 & 24 & 0 \\
\hline Stage $3 b$ & 381 & 304 & 258 & 206 & 103 & 0 \\
\hline Stage 4 & 444 & 330 & 270 & 203 & 104 & 1 \\
\hline Stage 5 & 196 & 100 & 69 & 43 & 13 & 1 \\
\hline
\end{tabular}

urology, obstetrics, and gynecology [12]; other two include benign neoplasm, trauma, emergency, hematologic disease, psychiatric, and neurological. $C K D$ chronic kidney disease, $D M$ diabetes mellitus

the incidence of hospitalization between CKD patients and the general population but also differences between large nephrology hospitals and the general population. At large 
(a)

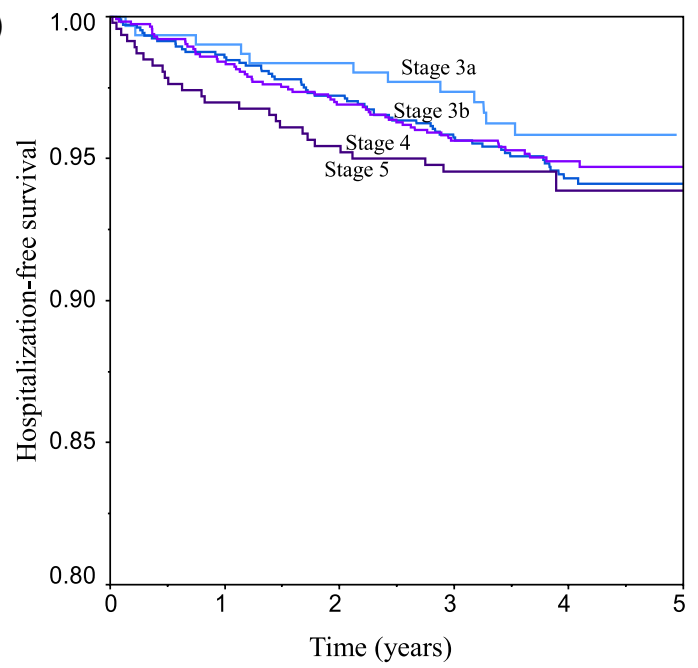

Survival rate

\begin{tabular}{|c|c|c|c|c|c|c|}
\hline Stage $3 \mathrm{a}$ & 100.0 & 99.0 & 98.4 & 97.4 & 95.8 & - \\
\hline Stage $3 b$ & 100.0 & 98.6 & 97.2 & 95.7 & 94.3 & 94.1 \\
\hline Stage 4 & 100.0 & 98.4 & 96.9 & 95.6 & 94.9 & 94.7 \\
\hline Stage 5 & 100.0 & 97.0 & 95.4 & 94.5 & 93.9 & 93.9 \\
\hline \multicolumn{7}{|c|}{ Number at risk } \\
\hline Stage $3 a$ & 307 & 302 & 299 & 273 & 165 & 0 \\
\hline Stage $3 b$ & 1047 & 1026 & 1008 & 925 & 600 & 1 \\
\hline Stage 4 & 1145 & 1111 & 1087 & 995 & 574 & 6 \\
\hline Stage 5 & 467 & 444 & 435 & 384 & 119 & 4 \\
\hline
\end{tabular}

(b)

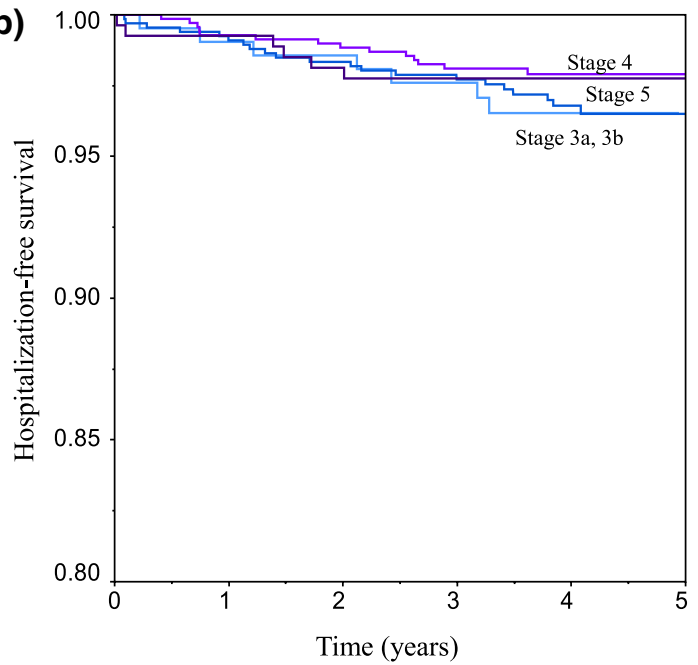

Survival rate

\begin{tabular}{|c|c|c|c|c|c|c|}
\hline \\
\hline Stage $3 a$ & 100.0 & 99.1 & 98.6 & 97.6 & 96.5 & - \\
\hline Stage $3 b$ & 100.0 & 99.1 & 98.3 & 97.7 & 96.8 & - \\
\hline Stage 4 & 100.0 & 99.3 & 98.8 & 98.1 & 97.9 & 97.9 \\
\hline Stage 5 & 100.0 & 99.3 & 98.1 & 97.8 & 97.8 & 97.8 \\
\hline \multicolumn{7}{|c|}{ Number at risk } \\
\hline Stage $3 a$ & 211 & 207 & 205 & 188 & 115 & 0 \\
\hline Stage $3 b$ & 666 & 655 & 648 & 601 & 400 & 0 \\
\hline Stage 4 & 701 & 685 & 678 & 627 & 370 & 4 \\
\hline Stage 5 & 271 & 264 & 261 & 232 & 84 & 2 \\
\hline
\end{tabular}

\section{(c)}

(c) $1.00 \sqrt{\frac{4}{4}}$

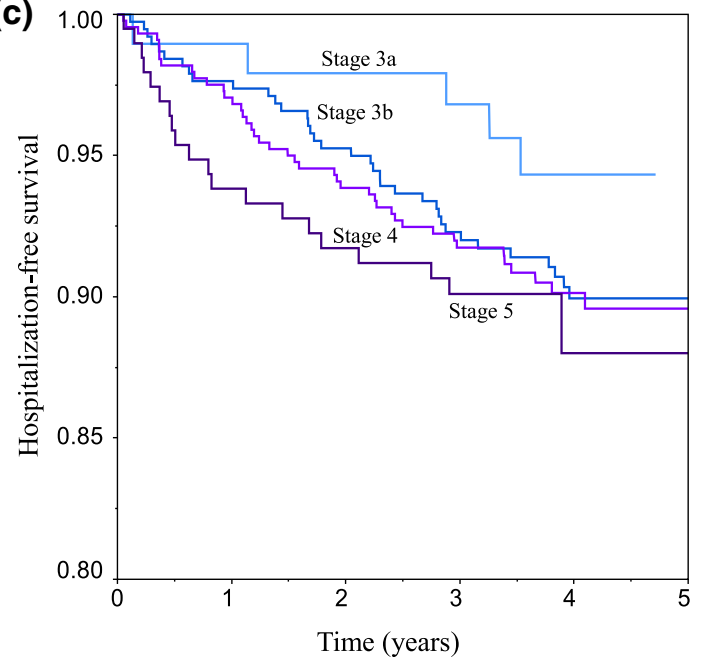

Survival rate

\begin{tabular}{|c|c|c|c|c|c|c|}
\hline Stage $3 a$ & 100.0 & 99.0 & 97.9 & 96.8 & 94.3 & - \\
\hline Stage $3 b$ & 100.0 & 97.6 & 95.3 & 92.3 & 90.0 & 90.0 \\
\hline Stage 4 & 100.0 & 97.1 & 93.9 & 91.7 & 90.1 & 89.6 \\
\hline Stage 5 & 100.0 & 93.8 & 91.7 & 90.1 & 88.0 & 88.0 \\
\hline \multicolumn{7}{|c|}{ Number at risk } \\
\hline Stage $3 a$ & 96 & 95 & 94 & 85 & 50 & 0 \\
\hline Stage $3 b$ & 381 & 371 & 360 & 324 & 200 & 1 \\
\hline Stage 4 & 444 & 426 & 409 & 368 & 204 & 2 \\
\hline Stage 5 & 196 & 180 & 174 & 152 & 35 & 2 \\
\hline
\end{tabular}

Fig. 4 Kaplan-Meier survival curves for the first hospitalization due to CVD. a CKD stage. b CKD stage and DM (-). c CKD stage and DM (+). Events included ischemic heart diseases and cerebral infarction from [06] heart diseases and [05] diseases of the circulatory system [11]. Other one includes otorhinolaryngology, dermatology, orthopedic conditions including bone and muscle, urology, obstetrics, and gynecology [12]; other two include benign neoplasm, trauma, emergency, hematologic disease, psychiatric, and neurological. CKD chronic kidney disease, $C V D$ cardiovascular disease, $D M$ diabetes mellitus 
hospitals, patients have the advantage of receiving treatment optimized to their disease state. Therefore, the incidence of hospitalization may be lower than that in ordinary hospitals. Third, proper evaluation of CKD during maintenance period must be based on the comparison between CKD-JAC group and general population which meets inclusion and exclusion criteria of CKD-JAC. However, it was impossible to do that, so we used patient survey data as the substitute. Even though the patient survey population was matched to the CKD-JAC population using sex, age, and person-years of observation as adjustment factors, these data contain both patients who get hospitalized at a certain day and those who have been hospitalized on the same day. That means we may overestimate the incidence of hospitalization in general population. In addition, more and more patients over 75 years old have started dialysis in recent years [25]. These aged people have higher risk for hospitalization than younger generation under 75 years old, not only for ESKD but also various aging conditions. To evaluate the risk of CKD more precisely, these elderly patients should have included to our study. Further study is expected for evaluating CKD on elderly people.

These might result in underestimation of the overall extent of hospitalization of CKD patients. However, we believe that the magnitude of differences described in this paper is sufficient to justify the generalizability of our results.

As for hospitalization durations, they are likely to be affected by the medical assurance system in Japan, so other suitable studies to evaluate them will be needed.

In addition to validating the previously observed increased risk for CVD and ESKD, this study clearly shows the extreme vulnerability of CKD patients to many other diseases. These data are highly valuable for predicting prognoses in CKD patients, in addition, for identifying high-risk population among CKD patients.

Acknowledgements This study was conducted by the principal investigators at the following medical centers: Japan Community Health Care Organization Sendai Hospital (Miyagi), JA Toride Medical Center (Ibaraki), Jichi Medical University (Tochigi), Tokyo Women's Medical University Hospital (Tokyo), St. Luke's International Hospital (Tokyo), Showa University Hospital (Tokyo), Showa University Yokohama Northern Hospital (Kanagawa), Showa University Fujigaoka Hospital (Kanagawa), Gifu Prefectural General Medical Center (Gifu), Kasugai Municipal Hospital (Aichi), Tosei General Hospital (Aichi), Osaka University Hospital (Osaka), Osaka General Medical Center (Osaka), Osaka City General Hospital (Osaka), Kurashiki Central Hospital (Okayama), Fukuoka Red Cross Hospital (Fukuoka), and Iizuka Hospital (Fukuoka)

\section{Compliance with ethical standards}

Conflict of interest SI and TK received a research support grant from Kyowa Hakko Kirin; KN received a research support grant from Kyowa Hakko Kirin; TA received consultancy and lecture fees from Kyowa Hakko Kirin; SM received lecture fees from Kyowa Hakko Kirin; HM received speaker honoraria from Kyowa Hakko Kirin. The other authors have no conflicts of interest to declare. This study was supported by research funds with no restriction on publication from $\mathrm{Ky}$ owa Hakko Kirin Co., Ltd.

Ethical Approval All procedures performed in studies involving human participants were in accordance with the ethical standards of the institutional and/or national research committee at which the studies were conducted and with the 1964 Helsinki Declaration and its later amendments or comparable ethical standards.

Informed Consent Informed consent was not required as this study analyzes previously published data.

Open Access This article is distributed under the terms of the Creative Commons Attribution 4.0 International License (http://creativeco mmons.org/licenses/by/4.0/), which permits unrestricted use, distribution, and reproduction in any medium, provided you give appropriate credit to the original author(s) and the source, provide a link to the Creative Commons license, and indicate if changes were made.

\section{References}

1. National Kidney Foundation. K/DOQI clinical practice guidelines for chronic kidney disease: evaluation, classification, and stratification. Am J Kidney Dis. 2002;39:1-266.

2. Radhakrishnan J, Remuzzi G, Saran R, Williams DE, Rios-Burrows N, Powe N, CDC-CKD Surveillance Team, Brück K, Wanner C, Stel VS, European CKD Burden Consortium. Venuthurupalli SK, Hoy WE, Healy HG, Salisbury A, Fassett RG; CKD.QLD group, O'Donoghue D, Roderick P, Matsuo S, Hishida A, Imai E, Iimuro $S$. Taming the chronic kidney disease epidemic: a global view of surveillance efforts. Kidney Int. 2014; 86:246-50.

3. Denker M, Boyle S, Anderson AH, Chen J, Fink JC, Flack J, Go AS, Horwitz E, Hsu CY, Kusek JW, Lash JP, Navaneethan S, Ojo AO, Rahman M, Steigerwalt SP, Townsend RR, Feldman HI. Chronic renal insufficiency cohort study investigators. chronic renal insufficiency cohort study (CRIC): overview and summary of selected findings. Clin J Am Soc Nephrol. 2015;10:2073-83.

4. Rahman M, Xie D, Feldman HI, Go AS, He J, Kusek JW, Lash J, Miller ER 3rd, Pan Q, Seliger SL, Steigerwalt S, Townsend RR. CRIC study investigators. Association between chronic kidney disease progression and cardiovascular disease: results from the CRIC study. Am J Nephrol. 2014;40:399-407.

5. Imai E, Matsuo S, Makino H, Watanabe T, Akizawa T, Nitta K, Iimuro S, Ohashi Y, Hishida A, CKD-JAC Study Group. Chronic kidney disease Japan cohort (CKD-JAC) study: design and methods. Hypertens Res. 2008;31:1101-7.

6. Imai E, Matsuo S, Makino H, Watanabe T, Akizawa T, Nitta K, Iimuro S, Ohashi Y, Hishida A. Chronic kidney disease Japan cohort study: baseline characteristics and factors associated with causative diseases and renal function. Clin Exp Nephrol. 2010;14:558-70.

7. Tanaka K, Watanabe T, Takeuchi A, Ohashi Y, Nitta K, Akizawa T, Matsuo S, Imai E, Makino H, Hishida A. CKD-JAC investigators. Cardiovascular events and death in Japanese patients with chronic kidney disease. Kidney Int. 2017;91:227-34.

8. Inaguma D, Imai E, Takeuchi A, Ohashi Y, Watanabe T, Nitta K, Akizawa T, Matsuo S, Makino H, Hishida A, Chronic Kidney Disease Japan Cohort Study Group. Risk factors for CKD progression in Japanese patients: findings from the chronic kidney disease Japan cohort (CKD-JAC) study. Clin Exp Nephrol. 2017;21:446-56. 
9. Sarnak MJ, Levey AS, Schoolwerth AC, Coresh J, Culleton B, Hamm LL, McCullough PA, Kasiske BL, Kelepouris E, Klag MJ, Parfrey P, Pfeffer M, Raij L, Spinosa DJ, Wilson PW, American Heart Association Councils on Kidney in Cardiovascular Disease. High blood pressure research, clinical cardiology, and epidemiology and prevention. Kidney disease as a risk factor for development of cardiovascular disease: a statement from the American Heart Association Councils on Kidney in Cardiovascular Disease, High Blood Pressure Research, Clinical Cardiology, and Epidemiology and Prevention. Circulation. 2003;108:2154-69.

10. Yamagata K, Ishida K, Sairenchi T, Takahashi H, Ohba S, Shiigai T, Narita M, Koyama A. Risk factors for chronic kidney disease in a community-based population: a 10-year follow-up study. Kidney Int. 2007;71:159-66.

11. Iseki K, Oshiro S, Tozawa M, Ikemiya Y, Fukiyama K, Takishita $\mathrm{S}$. Prevalence and correlates of diabetes mellitus in a screened cohort in Okinawa, Japan. Hypertens Res. 2002;25:185-90.

12. Muntner P, Coresh J, Smith JC, Eckfeldt J, Klag MJ. Plasma lipids and risk of developing renal dysfunction: the atherosclerosis risk in communities study. Kidney Int. 2000;58:293-301.

13. Schaeffner ES, Kurth T, Curhan GC, Glynn RJ, Baigent C, Buring JE, Gaziano JM. Cholesterol and the risk of renal dysfunction in apparently healthy men. J Am Soc Nephrol. 2003;14:2084-91.

14. Masakane I, Nakai S, Ogata S, Kimata N, Hanafusa N, Hamano T, Wakai K, Wada A, Nitta K. An Overview of Regular Dialysis Treatment in Japan (As of 31 December 2013). Ther Apher Dial. 2015;19(6):540-74.

15. Mix TC, St Peter WL, Ebben J, Xue J, Pereira BJ, Kausz AT, Collins AJ. Hospitalization during advancing chronic kidney disease. Am J Kidney Dis. 2003;42:972-81.

16. Go AS, Chertow GM, Fan D, McCulloch CE, Hsu CY. Chronic kidney disease and the risks of death, cardiovascular events, and hospitalization. N Engl J Med. 2004;351:1296-305.

17. Nishikawa $\mathrm{K}$, Takahashi $\mathrm{K}$, Yamada R, Kinaga T, Masato M, Yamamoto M. Influence of chronic kidney disease on hospitalization, chronic dialysis, and mortality in Japanese men: a longitudinal analysis. Clin Exp Nephrol. 2017;21:316-23.
18. Pisoni RL, Gillespie BW, Dickinson DM, Chen K, Kutner MH, Wolfe RA. The dialysis outcomes and practice patterns study (DOPPS): design, data elements, and methodology. Am J Kidney Dis. 2004;44:7-15.

19. Ministry of Health Labour and Welfare. International statistical classification of diseases and related health problems (ICD) - 10 Japanese edition 2003. http://www.mhlw.go.jp/toukei/sippei/. Accessed 30 Aug 2018.

20. Ministry of Health Labour and Welfare. Summary of patient survey. http://www.mhlw.go.jp/english/database/db-hss/sps_2008. html. Accessed 30 Aug 2018.

21. Muntner P, He J, Hamm L, Loria C, Whelton PK. Renal Insufficiency and subsequent death resulting from cardiovascular disease in the United States. J Am Soc Nephrol. 2002;13:745-53.

22. Keith DS, Nichols GA, Gullion CM, Brown JB, Smith DH. Longitudinal follow-up and outcomes among a population with chronic kidney disease in a large managed care organization. Arch Intern Med. 2004;164:659-63.

23. Manjunath G, Tighiouart H, Ibrahim H, MacLeod B, Salem DN, Griffith JL, Coresh J, Levey AS, Sarnak MJ. Level of kidney function as a risk factor for atherosclerotic cardiovascular outcomes in the community. J Am Coll Cardiol. 2003;41:47-55.

24. Ninomiya T, Kiyohara Y, Kubo M, Tanizaki Y, Doi Y, Okubo K, Wakugawa Y, Hata J, Oishi Y, Shikata K, Yonemoto K, Hirakata $\mathrm{H}$, Iida M. Chronic kidney disease and cardiovascular disease in a general Japanese population: the Hisayama Study. Kidney Int. 2005;68:228-36.

25. An Overview of Regular Dialysis Treatment in Japan. https:// www.jsdt.or.jp/english/2426.html. Accessed 15 Feb 2019.

Publisher's Note Springer Nature remains neutral with regard to jurisdictional claims in published maps and institutional affiliations. 